\title{
Pattern Synthesis of Narrowband Conformal Arrays Using Iterative Second-Order Cone Programming
}

\author{
K. M. Tsui and S. C. Chan, Member, IEEE
}

\begin{abstract}
A new design method is proposed for the power or shaped beam pattern synthesis problem of narrowband conformal arrays, where only the magnitude response is specified. The proposed method iteratively linearizes the non-convex power pattern function to obtain a convex subproblem in the design variables, which can be solved optimally using second-order cone programming (SOCP). In addition, a wide variety of magnitude constraints such as non-convex lower bound magnitude constraints can be incorporated. An efficient technique for determining a reasonably good initial guess to the problem is also proposed to further improve the reliability of the method. Computer simulations show that the initial guesses so obtained converge to satisfactory solutions while satisfying various prescribed magnitude constraints. Design results show that the performance of the proposed method is comparable to the optimal solution previously obtained for uniform linear arrays with isotropic elements. Moreover, we show by means of examples that the proposed method is also applicable to general non-convex power pattern synthesis problems involving arbitrary array geometries, arbitrary polarization characteristics and mutual coupling effect.
\end{abstract}

Index Terms-Conformal antenna arrays, mutual coupling, narrowband design, polarization, power pattern synthesis, second-order cone programming, shaped beam pattern synthesis.

\section{INTRODUCTION}

$\mathbf{T}$ HE analysis and design of conformal arrays have received considerable attention because of their flexibility in attaching to arbitrary surface of vehicles and aircrafts to save space and capability of offering wide angular coverage and avoiding boresight error, etc [1]. Unlike conventional arrays with regular geometry, the element excitations or factors of conformal arrays have to be designed by optimization techniques. In the general synthesis problem of narrowband conformal arrays, the complex element excitations are optimized so that the resulting radiation pattern satisfies a desired beam shape possibly with a specified tolerance. Sometimes it is also necessary to consider the polarization characteristics and directivity of individual element, which depends on the orientation of the elements. Fortunately, the radiation pattern is a linear function of the complex element excitations. Hence if both magnitude and phase responses of the desired beam shape are specified,

Manuscript received July 17, 2009; revised November 11, 2009; accepted January 11, 2010. Date of publication March 29, 2010; date of current version June 03, 2010.

The authors are with the Department of Electrical and Electronic Engineering, The University of Hong Kong, Pokfulam, Hong Kong (email: kmtsui@eee. hku.hk; scchan@eee.hku.hk).

Digital Object Identifier 10.1109/TAP.2010.2046865 the overall problem is convex and is closely related to conventional filter design problems [2], [3]. This has attracted much interest in using efficient convex optimization methods to solve various beam pattern synthesis problems [4]-[6]. An important advantage of convex programming is its ability to satisfy multiple objectives expressed in terms of a set of linear and convex quadratic constraints. Moreover, the optimality of the solution, if it exists, is guaranteed. Efficient pattern synthesis techniques are useful in predicting the radiation patterns and configurations of the target array during initial design stage, or further improving its performance when real measurements are available.

On the other hand, the pattern specifications are very often expressed in power (or decibels) and the pattern phase is generally unimportant, say in applications such as radar and communication systems. Beam shaping, as in cosecant squared patterns, is a typical example where phase response is of no concern. This kind of problem is known as power or shaped beam pattern synthesis problem, where only magnitude response is specified [7]-[13]. Although the resulting problem is in general non-convex and more than one solution to the synthesis problem may exist, additional design freedom is available and hence the performance of the synthesized result is usually better than the convex beam pattern synthesis problem mentioned above [13]. It should be noted that the power pattern synthesis of uniform linear arrays with isotropic elements is the only exception that convex programming is applicable [7], [8]. By using the autocorrelation sequence representation of the excitations, it is possible to find an optimal power pattern in this simple case. For practical implementation, spectral factorization is further required to extract the corresponding complex excitations from the optimal power pattern. However, these approaches are not suitable for the general array geometry and directive elements. To cope with the general non-convex power pattern synthesis problem, various optimization methods have also been proposed [9]-[13]. The stochastic optimization methods, including genetic algorithm [9], particle swarm optimization [10] and simulated annealing [11], are very general and flexible frameworks, but their computational complexities are rather high because the searching methods involved are random in nature. The semidefinite programming (SDP) method in [12] focused only on nonuniform linear arrays with isotropic elements, and it relaxed the non-convex lower bound constraint and solved a series of subproblems using SDP. Whereas the iterative least-squares method in [13] employed a virtual phase response obtained from the previous iteration and solved a least-squares pattern synthesis problem successively to improve the synthesized result. However, it is 
somewhat difficult to precisely control the beam pattern and incorporate inequality constraints, such as prescribed lower bound constraints.

In this paper, we propose a general design method for the power beam pattern synthesis problem using iterative second-order cone programming (SOCP). It extends the iterative methods proposed in [14] and [15] for the design of IIR and IIR frequency-response masking digital filters to the array pattern synthesis problem. Although these filter design problems are highly nonlinear, it is shown that the iterative SOCP approach is capable of finding better solution than conventional methods in digital filter design community. The basic idea of the proposed approach is to linearize the power pattern function (i.e., the magnitude square of the radiation pattern) of the conformal array in a neighborhood of the complex excitations in each iteration. By so doing, the original non-convex problem is relaxed to a series of convex SOCP subproblems with respect to the corresponding update vectors around the previous iterates. Hence, the advantages of previously mentioned convex programming can be utilized. For example, a wide variety of magnitude constraints can be incorporated easily in the problem formulation. To ensure the accuracy of the linear approximation, a norm constraint on the update vector is further imposed under the SOCP framework. The idea is similar to the conventional trust region method [16].

As the original problem is generally non-convex, a reasonably good initial guess is very important to achieve a good solution. To this end, we propose a simple way to obtain an initial guess, which satisfies all the constraints specified in the original non-convex power beam pattern synthesis problem. Since non-convex constraints are usually involved, it is not straightforward to find such initial guess. Fortunately, as mentioned earlier, if a suitable phase response is known, the original problem can be approximated as a convex programming problem. Therefore, we propose to employ a virtual phase response, which is the average phase of all the elements, so that the initial guess satisfying all the constraints can be obtained by solving a convex programming problem. In the simple case of uniform linear array with isotropic elements, design result of a cosecant squared pattern shows that the initial guess so obtained converges to a solution which is comparable to the optimal solution found using the convex optimization method in [7]. This suggests that the proposed approach with the above choice of the initial guess is reliable and efficient in obtaining a near optimal result. A more complicated example of hemispherical array with circular polarization is also given to demonstrate the flexibility and effectiveness of the proposed approach in satisfying various magnitude constraints for different polarization components. Moreover, the proposed method can be readily extended to solve pattern synthesis problems including the effect of mutual coupling.

The paper is organized as follows: Section II is devoted to the general radiation pattern of conformal arrays with polarization. In Section III, the proposed iterative SOCP method and efficient method for determining the initial guess are presented. Design examples are given in Section IV. Finally, conclusion is drawn in Section V.

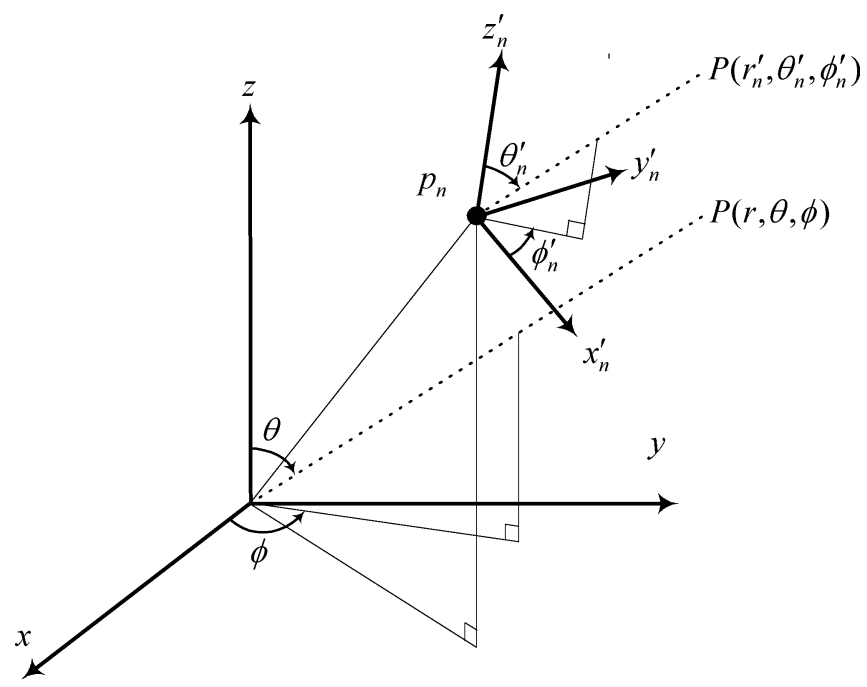

Fig. 1. Global and local coordinate systems.

\section{RADIATION PATTERN OF CONFORMAL ARRAY}

Consider a conformal array of $N$ elements located arbitrarily at a carrier surface. To determine the total far-field radiation pattern produced by the conformal array at a far-field point $P(r, \theta, \phi)$, it is more convenient to first consider the field of individual elements in their own coordinate systems and then transform the field back to the global coordinate system. Fig. 1 shows the local coordinate system $\left(x_{n}^{\prime}, y_{n}^{\prime}, z_{n}^{\prime}\right)$ of the $n$th element with origin at its position $p_{n}$, and the global coordinate system $(x, y, z)$. The element is pointed in the direction of the $z_{n}^{\prime}$-axis. The spherical coordinate of the common far-field point $P$ in the local coordinate system are $r_{n}^{\prime}, \theta_{n}^{\prime}$ and $\phi_{n}^{\prime}$. Hence, the field of the $n$th element with respect to its coordinate system is given by

$$
\boldsymbol{f}_{n}^{\prime}\left(\theta_{n}^{\prime}, \phi_{n}^{\prime}\right)=\hat{\boldsymbol{u}}_{\Theta_{n}^{\prime}} f_{\Theta_{n}^{\prime}}\left(\theta_{n}^{\prime}, \phi_{n}^{\prime}\right)+\hat{\boldsymbol{u}}_{\Phi_{n}^{\prime}} f_{\Phi_{n}^{\prime}}\left(\theta_{n}^{\prime}, \phi_{n}^{\prime}\right)
$$

where $\hat{\boldsymbol{u}}_{\Theta_{n}^{\prime}}=\left[\cos \left(\theta_{n}^{\prime}\right) \cos \left(\phi_{n}^{\prime}\right), \cos \left(\theta_{n}^{\prime}\right) \sin \left(\phi_{n}^{\prime}\right),-\sin \left(\theta_{n}^{\prime}\right)\right]^{T}$ and $\hat{\boldsymbol{u}}_{\Phi_{n}^{\prime}}=\left[-\sin \left(\phi_{n}^{\prime}\right), \cos \left(\phi_{n}^{\prime}\right), 0\right]^{T}$ are respectively the unit vectors in the $\theta_{n}^{\prime}$ and $\phi_{n}^{\prime}$ directions; and $f_{\Theta_{n}^{\prime}}\left(\theta_{n}^{\prime}, \phi_{n}^{\prime}\right)$ and $f_{\Phi_{n}^{\prime}}\left(\theta_{n}^{\prime}, \phi_{n}^{\prime}\right)$ are respectively the element pattern functions of the $\theta_{n}^{\prime}$ and $\phi_{n}^{\prime}$ components. Note these element pattern functions can either be described by a suitable model for simulation purpose, or obtained from measurements or EM simulations so as to take the actual radiation properties of real antenna systems and their mutual coupling into account [24]. Other effects such as measurement errors and deviation in radiation characteristics due to the feeding and supporting structures etc are not considered in our formulation. Regarding real examples and physical realizations of antenna elements, interested readers are referred to [25]-[32] for more details.

Since each element has its own position and orientation, coordinate transformation is required so that all the elements' components can be superimposed at the far-field point $P(r, \theta, \phi)$ in the global coordinate system $(x, y, z)$ [17], [18]. More precisely, we want to express $\theta_{n}^{\prime}, \phi_{n}^{\prime}, \hat{\boldsymbol{u}}_{\Theta_{n}^{\prime}}$ and $\hat{\boldsymbol{u}}_{\Phi_{n}^{\prime}}$ in terms of $\theta, \phi, \hat{\boldsymbol{u}}_{\Theta}$ 
and $\hat{\boldsymbol{u}}_{\Phi}$, where the unit vectors $\hat{\boldsymbol{u}}_{\ominus}$ and $\hat{\boldsymbol{u}}_{\Phi}$ are defined similarly as $\hat{\boldsymbol{u}}_{\Theta_{n}^{\prime}}$ and $\hat{\boldsymbol{u}}_{\Phi_{n}^{\prime}}$ except that $\theta_{n}^{\prime}$ and $\phi_{n}^{\prime}$ are respectively replaced by $\theta$ and $\phi$ in global coordinate system.

In general, the coordinate transformation from global coordinate system $(x, y, z)$ to local coordinate system $\left(x_{n}^{\prime}, y_{n}^{\prime}, z_{n}^{\prime}\right)$ can be characterized by a rotation matrix $\boldsymbol{M}_{n}$ and inversely by $\boldsymbol{M}_{n}^{-1}=\boldsymbol{M}_{n}^{T}$. Very often, three separate axis rotations are sufficient to fully describe the position and orientation of the element. Interested readers are referred to [17] and [18] for more details. The relation of the former coordinate transformation is given by

$$
\left[x_{n}^{\prime}, y_{n}^{\prime}, z_{n}^{\prime}\right]^{T}=\boldsymbol{M}_{n}[x, y, z]^{T} .
$$

Using this result, $\theta_{n}^{\prime}$ and $\phi_{n}^{\prime}$ can be expressed as

$$
\cos \left(\theta_{n}^{\prime}\right)=z_{r_{n}^{\prime}} \text { and } \tan \left(\phi_{n}^{\prime}\right)=\frac{y_{r_{n}^{\prime}}}{x_{r_{n}^{\prime}}}
$$

where $\hat{\boldsymbol{u}}_{R_{n}^{\prime}}=\left[x_{r_{n}^{\prime}}, y_{r_{n}^{\prime}}, z_{r_{n}^{\prime}}\right]^{T}=\boldsymbol{M}_{n} \hat{\boldsymbol{u}}_{R}$; and $\hat{\boldsymbol{u}}_{R}=$ $[\sin (\theta) \cos (\phi), \sin (\theta) \sin (\phi), \cos (\theta)]^{T}$ is the unit vector in radial direction.

Next, we consider the polarization components in (2-1) after coordinate transformation. The basic idea is to first transform the polarization components into the global coordinate system and then project these rotated polarization components onto the polarization components in the global coordinate system. Consequently, the field of the $n$th element with respect to the global coordinate system can be expressed as

$$
\begin{aligned}
& \boldsymbol{f}_{n}\left(\theta_{n}^{\prime}, \phi_{n}^{\prime}\right) \\
& =\hat{\boldsymbol{u}}_{\Theta}\left\{\hat{\boldsymbol{u}}_{\Theta}^{T} \boldsymbol{M}_{n}^{T} \boldsymbol{f}_{n}^{\prime}\left(\theta_{n}^{\prime}, \phi_{n}^{\prime}\right)\right\}+\hat{\boldsymbol{u}}_{\Phi}\left\{\hat{\boldsymbol{u}}_{\Phi}^{T} \boldsymbol{M}_{n}^{T} \boldsymbol{f}_{n}^{\prime}\left(\theta_{n}^{\prime}, \phi_{n}^{\prime}\right)\right\} .
\end{aligned}
$$

Hence, according to (2-1) - (2-4), the total far-field radiation pattern of the $N$-element array can be written as

$$
\begin{aligned}
\boldsymbol{E}(\theta, \phi) & =\hat{\boldsymbol{u}}_{\Theta} E_{\Theta}(\theta, \phi)+\hat{\boldsymbol{u}}_{\Phi} E_{\Phi}(\theta, \phi) \\
E_{\Theta}(\theta, \phi) & =\sum_{n=1}^{N} a_{n} e^{j k_{0} \boldsymbol{p}_{n}^{T} \hat{\boldsymbol{u}}_{R}} q_{n, \Theta}(\theta, \phi)
\end{aligned}
$$

and

$$
E_{\Phi}(\theta, \phi)=\sum_{n=1}^{N} a_{n} e^{j k_{0} \boldsymbol{p}_{n}^{T} \hat{\boldsymbol{u}}_{R}} q_{n, \Phi}(\theta, \phi)
$$

where $q_{n, \Theta}(\theta, \phi)=\hat{\boldsymbol{u}}_{\Theta}^{T} \boldsymbol{M}_{n}^{T} \boldsymbol{f}_{n}^{\prime}\left(\theta_{n}^{\prime}, \phi_{n}^{\prime}\right) ; q_{n, \Phi}(\theta, \phi)=$ $\hat{\boldsymbol{u}}_{\Phi}^{T} \boldsymbol{M}_{n}^{T} \boldsymbol{f}_{n}^{\prime}\left(\theta_{n}^{\prime}, \phi_{n}^{\prime}\right) ; a_{n}$ is the complex excitation amplitude of the $n$th element; $k_{0}=2 \pi / \lambda$ is the wave number with wavelength $\lambda$; and $\boldsymbol{p}_{n}=\left[x_{n}, y_{n}, z_{n}\right]^{T}$ is the position vector of the $n$th element. More generally, if we denote $\hat{\boldsymbol{u}}_{c o}$ and $\hat{\boldsymbol{u}}_{c p}$ respectively as the co- and cross-polarization unit vectors and assume $\hat{\boldsymbol{u}}_{c o}$ is orthogonal to $\hat{\boldsymbol{u}}_{c p}$, then the corresponding polarization components can be expressed as:

$$
E_{c o}(\theta, \phi)=\hat{\boldsymbol{u}}_{c o}^{T} \boldsymbol{E}(\theta, \phi) \text { and } E_{c p}(\theta, \phi)=\hat{\boldsymbol{u}}_{c p}^{T} \boldsymbol{E}(\theta, \phi)
$$

which are also linear functions of $a_{n}$. On the other hand, if either one of the polarization components is considered, the corresponding radiation pattern is given by:

$$
E_{\Psi}(\theta, \phi)=\sum_{n=1}^{N} a_{n} e^{j k_{0} \boldsymbol{p}_{n}^{T} \hat{\boldsymbol{u}}_{R}} q_{n, \Psi}(\theta, \phi) .
$$

Here we assume the subscripts (2-6) - (2-8) are collectively represented by $\Psi$ for notation simplicity.

\section{POWER PATTERN SYNTHESIS}

\section{A. General Problem Definition}

In pattern synthesis of a conformal array, a commonly considered problem is to determine the element excitation $a_{n}$ such that the radiation pattern approximates a desired beam pattern:

$$
E_{\Psi}(\theta, \phi) \sim M_{\Psi}(\theta, \phi) e^{j p_{\Psi}(\theta, \phi)},(\theta, \phi) \in \Omega_{\Psi}
$$

where $M_{\Psi}(\theta, \phi)$ and $p_{\Psi}(\theta, \phi)$ are respectively the desired magnitude and phase responses of $E_{\Psi}(\theta, \phi)$, and $\Omega_{\Psi}$ is the region of interest. Note by definition $M_{\Psi}(\theta, \phi) \geq 0$ for $(\theta, \phi) \in \Omega_{\Psi}$. Without loss of generality, subsequent discussions are based on $E_{\Psi}(\theta, \phi)$ in (2-9). Similar idea can be directly applied to all kinds of polarization components defined in $(2-6)-(2-8)$. A typical pattern synthesis problem is to minimize the maximum absolute complex approximation error between $E_{\Psi}(\theta, \phi)$ and $M_{\Psi}(\theta, \phi) e^{j p_{\Psi}(\theta, \phi)}$. That is

$$
\min _{a_{n}} \max _{(\theta, \phi) \in \Omega_{\Psi}}\left|E_{\Psi}(\theta, \phi)-M_{\Psi}(\theta, \phi) e^{j p_{\Psi}(\theta, \phi)}\right| .
$$

Alternatively, the least squares (LS) error criterion [3] and the maximization of the partial directivity measure [19] are frequently considered. Since the desired phase response is given, the error response is a linear function of the complex excitations $a_{n}$. Consequently, all these optimization problems can be readily formulated as convex programming problem such as SOCP and SDP [4]-[6]. The main advantages of these approaches are that additional constraints can be easily incorporated, and the solution is guaranteed to be optimal if the overall problem is convex. For example, one may impose the magnitude constraint in the form of $\left|E_{\Psi}(\theta, \phi)\right| \leq U_{\Psi}(\theta, \phi)$ to shape the maximum value of the magnitude response according to a given function $U_{\Psi}(\theta, \phi)$.

A more challenging pattern synthesis problem is to approximate only a desired magnitude response, while keeping the de- 
sired phase unspecified. More precisely, one wishes to obtain the desired magnitude response as

$$
\left|E_{\Psi}(\theta, \phi)\right| \sim M_{\Psi}(\theta, \phi), \quad(\theta, \phi) \in \Omega_{\Psi}
$$

or equivalently, after squaring both sides of (3-3),

$$
\left|E_{\Psi}(\theta, \phi)\right|^{2} \sim M_{\Psi}^{2}(\theta, \phi),(\theta, \phi) \in \Omega_{\Psi}
$$

which is a commonly encountered problem in applications such as radar and communication systems. In fact, this problem is equivalent to conventional shaped beam or power pattern synthesis problem. The concept is intuitively simple, but the design problem is unfortunately non-convex in general due to the lack of the desired phase response.

In this paper, we shall focus on the following problem of power pattern synthesis based on minimax criterion

$$
\left.\min _{a_{n}} \max _{(\theta, \phi) \in \Omega_{\Psi}}|| E_{\Psi}(\theta, \phi)\right|^{2}-M_{\Psi}^{2}(\theta, \phi) \mid
$$

subject to the following constraint specifying only the bound of the desired magnitude response:

$$
L_{\Psi}^{2}(\theta, \phi) \leq\left|E_{\Psi}(\theta, \phi)\right|^{2} \leq U_{\Psi}^{2}(\theta, \phi), \quad(\theta, \phi) \in \Omega_{\Psi}
$$

where $L_{\Psi}(\theta, \phi)$ and $U_{\Psi}(\theta, \phi)$ are respectively the lower and upper bound functions of the desired magnitude response and $L_{\Psi}(\theta, \phi) \geq 0$. Obviously, neither the approximation problem in (3-5) nor the magnitude constraint in (3-6) is convex in nature due to the lower bound of $\left|E_{\Psi}(\theta, \phi)\right|^{2}$. Hence, the convex programming methods are not directly applicable.

It should be noted that there are previous attempts in solving a similar problem in (3-5) possibly with the constraint in (3-6) using convex programming [7], [8]. However, these approaches are only suitable for uniform linear array with isotropic elements (i.e., $q_{n, \Psi}(\theta, \phi)=1$ ). For more general cases considered in this paper, the synthesis problem is usually more difficult, and very often has to be solved by means of certain forms of problem approximation through relaxations and iterative methods such as the approaches in [12] and [13]. In [13], an iterative weighted least squares method was proposed to iteratively minimize the sum of squares of the approximation error, while the desired phase response is varied and updated according to previous iterates. It was found that if the desired phase response is appropriately chosen in each iteration, the final synthesized result is usually much better than the case where the desired phase response is fixed (i.e., the problem in (3-1)). A disadvantage of this method is that it is not straightforward to incorporate additional inequality constraints in the formulation.

\section{B. Problem Formulation}

In the subsequent subsections, we shall describe a general design method for solving the non-convex power pattern synthesis problem. The proposed method can be viewed as a variant of the iterative SOCP filter design methods proposed in [14] and [15], because we extend these methods to deal with real-valued power pattern function with complex variables. The main advantage of the proposed approach is that a wide variety of constraints can be incorporated because the subproblem in each iteration can be solved optimally using SOCP.

As an example, we consider the following constrained optimization problem

$$
\begin{aligned}
& \left.\min _{a_{n}} \max _{(\theta, \phi) \in \Omega_{\Psi, 1}}|| E_{\Psi}(\theta, \phi)\right|^{2}-M_{\Psi}^{2}(\theta, \phi) \mid \\
& \text { subject to } \\
& L_{\Psi}^{2}(\theta, \phi) \leq\left|E_{\Psi}(\theta, \phi)\right|^{2} \leq U_{\Psi}^{2}(\theta, \phi), \quad(\theta, \phi) \in \Omega_{\Psi, 2} \\
& \left|E_{\Psi}(\theta, \phi)\right|^{2} \geq V_{\Psi}^{2}(\theta, \phi), \quad(\theta, \phi) \in \Omega_{\Psi, 3}
\end{aligned}
$$

where $\Omega_{\Phi, 1}, \Omega_{\Phi, 2}$ and $\Omega_{\Phi, 3}$ represent three different regions of interest, and $V_{\Psi}(\theta, \phi) \geq 0$ is an additional lower bound function. Due to the lower bound constraints of $\left|E_{\Theta}(\theta, \phi)\right|^{2}$, the problem in (3-7) is in general a non-convex optimization problem. Moreover, it is a very general problem, which covers the most common types of non-convex objective function and constraints related to the magnitude-only specification in the power pattern synthesis problem. For simplicity, we do not consider convex objective function and constraints because they can similarly be handled without much difficulty. Now we introduce a slack variable $\varepsilon$ and reformulate (3-7) as

$$
\begin{aligned}
\min _{a_{n}, \varepsilon} \varepsilon & \\
\text { subject to } & \left.|| E_{\Psi}(\theta, \phi)\right|^{2}-M_{\Psi}^{2}(\theta, \phi) \mid \\
& \leq \varepsilon,(\theta, \phi) \in \Omega_{\Psi, 1} \\
L_{\Psi}^{2}(\theta, \phi) & \leq\left|E_{\Psi}(\theta, \phi)\right|^{2} \leq U_{\Psi}^{2}(\theta, \phi), \quad(\theta, \phi) \in \Omega_{\Psi, 2} \\
\left|E_{\Psi}(\theta, \phi)\right|^{2} & \geq V_{\Psi}^{2}(\theta, \phi), \quad(\theta, \phi) \in \Omega_{\Psi, 3} .
\end{aligned}
$$

For notation simplicity, the argument variables $(\theta, \phi)$ are dropped in subsequent derivations. First of all, we rewrite (2-9) more compactly in matrix form as

$$
E_{\Psi}=\boldsymbol{a}^{T} \boldsymbol{q}_{\Psi}
$$

where $\boldsymbol{a}=\left[a_{1}, \ldots, a_{N}\right]^{T}$ and $\boldsymbol{q}_{\Psi}=$ $\left[e^{j k_{0} \boldsymbol{p}_{1}^{T} \hat{\boldsymbol{u}}_{R}} q_{1, \Psi}, \ldots, e^{j k_{0} \boldsymbol{p}_{N}^{T} \hat{\boldsymbol{u}}_{R}} q_{N, \Psi}\right]^{T}$. Let $\boldsymbol{a}=\boldsymbol{a}_{R}+j \boldsymbol{a}_{I}$, and $\tilde{\boldsymbol{c}}_{\Psi}$ and $\tilde{\boldsymbol{s}}_{\Psi}$ be respectively real and imaginary parts of $\boldsymbol{q}_{\Psi}$. Then $E_{\Psi}$ can be rewritten as

$$
E_{\Psi}=\boldsymbol{h}^{T}\left\{\boldsymbol{c}_{\Psi}+j \boldsymbol{s}_{\Psi}\right\}
$$

where $\boldsymbol{h}=\left[\begin{array}{ll}\boldsymbol{a}_{R}^{T} & \boldsymbol{a}_{I}^{T}\end{array}\right]^{T}, \boldsymbol{c}_{\Psi}=\left[\begin{array}{ll}\tilde{\boldsymbol{c}}_{\Psi}^{T} & -\tilde{\boldsymbol{s}}_{\Psi}^{T}\end{array}\right]^{T}$ and $\boldsymbol{s}_{\Psi}=\left[\begin{array}{ll}\tilde{\boldsymbol{s}}_{\Psi}^{T} & \tilde{\boldsymbol{c}}_{\Psi}^{T}\end{array}\right]^{T}$. Further, we define the squared magnitude response as

$$
H_{\Psi}(\boldsymbol{h})=\left|E_{\Psi}\right|^{2}=E_{\Psi} E_{\Psi}^{*}=\boldsymbol{h}^{T} \boldsymbol{A}_{\Psi} \boldsymbol{h}
$$


where $\boldsymbol{A}_{\Psi}=\boldsymbol{c}_{\Psi} \boldsymbol{c}_{\Psi}^{T}+\boldsymbol{s}_{\Psi} \boldsymbol{s}_{\Psi}^{T}$ and $E_{\Psi}^{*}$ denote the complex conjugate of $E_{\Psi}$. Hence, the problem in (3-8) can be reformulated as

$$
\begin{aligned}
\min _{\boldsymbol{h}, \varepsilon} \varepsilon & \\
\text { subject to }\left|H_{\Psi}(\boldsymbol{h})-M_{\Psi}^{2}\right| & \leq \varepsilon, \quad(\theta, \phi) \in \Omega_{\Psi, 1} \\
L_{\Psi}^{2} \leq H_{\Psi}(\boldsymbol{h}) & \leq U_{\Psi}^{2}, \quad(\theta, \phi) \in \Omega_{\Psi, 2} \\
H_{\Psi}(\boldsymbol{h}) & \geq V_{\Psi}^{2}, \quad(\theta, \phi) \in \Omega_{\Psi, 3} .
\end{aligned}
$$

\section{Design Methodology}

The proposed iterative optimization procedure starts with a reasonably good initial guess $\boldsymbol{h}_{0}$. Suppose that after $k$ iterations, we arrive at a point $\boldsymbol{h}_{k}$. For a smooth function $H_{\Psi}(\boldsymbol{h})$ in a vicinity of $\boldsymbol{h}_{k}$, we consider a linear approximation of $H_{\Psi}\left(\boldsymbol{h}_{k}\right)$ as follows

$$
H_{\Psi}\left(\boldsymbol{h}_{k}+\boldsymbol{\delta}\right) \approx H_{\Psi}\left(\boldsymbol{h}_{k}\right)+\boldsymbol{g}_{\Psi}^{T}\left(\boldsymbol{h}_{k}\right) \boldsymbol{\delta}
$$

where $\boldsymbol{g}_{\Psi}\left(\boldsymbol{h}_{k}\right)=2 \boldsymbol{A}_{\Psi} \boldsymbol{h}_{k}$ is the gradient of $H_{\Psi}(\boldsymbol{h})$ with respect to $\boldsymbol{h}$, evaluated at $\boldsymbol{h}=\boldsymbol{h}_{k}$, and $\boldsymbol{\delta}$ is the linear update vector such that $\boldsymbol{h}_{k+1}=\boldsymbol{h}_{k}+\boldsymbol{\delta}$. Substituting $\boldsymbol{h}=\boldsymbol{h}_{k}+\boldsymbol{\delta}$ into the problem in (3-12) and using the linear approximation in (3-13), the new solution $\boldsymbol{h}_{k+1}$ can be obtained by solving $\boldsymbol{\delta}$. This process is repeated until the relative change of two successive solutions is insignificant or the maximum number of iteration is reached. Consequently, the problem in (3-12) can be approximated as

$$
\begin{aligned}
\min _{\boldsymbol{\delta}, \varepsilon} \varepsilon & \\
\text { subject to }-\varepsilon & \leq H_{\Psi}\left(\boldsymbol{h}_{k}\right)+\boldsymbol{g}_{\Psi}^{T}\left(\boldsymbol{h}_{k}\right) \boldsymbol{\delta}-M_{\Psi}^{2} \\
& \leq \varepsilon, \quad(\theta, \phi) \in \Omega_{\Psi, 1} \\
H_{\Psi}\left(\boldsymbol{h}_{k}\right)+\boldsymbol{g}_{\Psi}^{T}\left(\boldsymbol{h}_{k}\right) \boldsymbol{\delta} & \geq 0, \quad(\theta, \phi) \in \Omega_{\Psi, 1} \\
L_{\Psi}^{2} \leq H_{\Psi}\left(\boldsymbol{h}_{k}\right)+\boldsymbol{g}_{\Psi}^{T}\left(\boldsymbol{h}_{k}\right) \boldsymbol{\delta} & \leq U_{\Psi}^{2}, \quad(\theta, \phi) \in \Omega_{\Psi, 2} \\
H_{\Psi}\left(\boldsymbol{h}_{k}\right)+\boldsymbol{g}_{\Psi}^{T}\left(\boldsymbol{h}_{k}\right) \boldsymbol{\delta} & \geq V_{\Psi}^{2}, \quad(\theta, \phi) \in \Omega_{\Psi, 3} \\
\|\boldsymbol{\delta}\| & \leq \delta_{\max } .
\end{aligned}
$$

where $\delta_{\max }$ is a prescribed positive step size bound to ensure that the linear approximation in (3-13) is sufficiently accurate. This can also be viewed as a trust region method by restricting the norm of $\boldsymbol{\delta}$. Note, to ensure that $H_{\Psi}(\boldsymbol{h})$ is always positive by definition, an extra constraint $H_{\Psi}\left(\boldsymbol{h}_{k}\right)+\boldsymbol{g}_{\Psi}^{T}\left(\boldsymbol{h}_{k}\right) \boldsymbol{\delta} \geq 0$ for $(\theta, \phi) \in \Omega_{\Psi, 1}$ is imposed, yet it is active only when $M_{\Psi}^{2}-\varepsilon \leq 0$. Since the first three constraints in (3-14) are linear inequalities and the last constraint is a convex quadratic constraint, the overall problem is convex, which can be solved using either SOCP with discretization of $\theta$ and $\phi$ [7], or SDP without discretization of $\theta$ and $\phi$ [8]. In this paper, we only focus on the former approach. Interested readers are referred to [7] for the pros and cons of these two approaches.

By defining the set $S_{d}$

$$
S_{d}=S_{\Theta, d} \times S_{\Phi, d}
$$

where $S_{\Theta, d}=\left\{\theta_{u}, u=1, \ldots, N_{\Theta}\right\}$ and $S_{\Phi, d}=\left\{\phi_{v}, v=\right.$ $\left.1, \ldots, N_{\Phi}\right\}$ are respectively two sets containing $N_{\Theta}$ and $N_{\Phi}$ uniformly sampled points of $\theta$ and $\phi$ on the whole range of interest, the above problem can be casted as the following SOCP

$$
\begin{aligned}
\min _{\boldsymbol{\delta}, \varepsilon} \varepsilon & \\
\text { subject to }-\varepsilon & \leq H_{\Psi}\left(\boldsymbol{h}_{k}\right)+\boldsymbol{g}_{\Psi}^{T}\left(\boldsymbol{h}_{k}\right) \boldsymbol{\delta}-M_{\Psi}^{2} \\
& \leq \varepsilon, \quad\left(\theta_{u}, \phi_{v}\right) \in \Omega_{\Phi, 1} \cap S_{d} \\
H_{\Psi}\left(\boldsymbol{h}_{k}\right)+\boldsymbol{g}_{\Psi}^{T}\left(\boldsymbol{h}_{k}\right) \boldsymbol{\delta} & \geq 0, \quad\left(\theta_{u}, \phi_{v}\right) \in \Omega_{\Phi, 1} \cap S_{d} \\
L_{\Psi}^{2} \leq H_{\Psi}\left(\boldsymbol{h}_{k}\right)+\boldsymbol{g}_{\Psi}^{T}\left(\boldsymbol{h}_{k}\right) \boldsymbol{\delta} & \leq U_{\Psi}^{2}, \quad\left(\theta_{u}, \phi_{v}\right) \in \Omega_{\Phi, 2} \cap S_{d} \\
H_{\Psi}\left(\boldsymbol{h}_{k}\right)+\boldsymbol{g}_{\Psi}^{T}\left(\boldsymbol{h}_{k}\right) \boldsymbol{\delta} & \geq V_{\Psi}^{2}, \quad\left(\theta_{u}, \phi_{v}\right) \in \Omega_{\Phi, 3} \cap S_{d} \\
\|\boldsymbol{\delta}\| & \leq \delta_{\max } .
\end{aligned}
$$

Consequently, $\boldsymbol{\delta}$ can be solved optimally in each iteration. Moreover, additional linear equality and convex inequality constraints can be easily incorporated in the above formulation.

It should be noted that the algorithm presented above converges to a local solution due to the linear approximation in (3-13). Therefore, the determination of a reasonably good initial guess, which governs the quality of the solution, is important. In Section III-D, we shall introduce an efficient technique to find such an initial guess. As illustrated subsequently in a representative example to be presented in Section IV-A for the synthesis of a uniform linear array, the proposed algorithm with the initial guess so obtained is capable of finding a solution that is close to the optimal solution obtained using the method in [7]. The good convergence performance of the proposed algorithm is largely attributed to the global convergence of individual subproblem and the sufficiently small norm bound of the update vector, as suggested in [14] and [15]. The proposed algorithm might also be viewed as a SOCP-based trust region method with simplified update steps [16]. A clear advantage of the proposed method is that the step size and step direction characterized by the norm bound constraint in each convex subproblem would be efficiently and optimally handled by well developed interior-point methods, see [17], [18] and references therein.

Also, a possible way to speed up the convergence is to adaptively adjust the norm bound in each iteration. Additional control mechanism is required to make a good tradeoff between the accuracy of the linearization and the convergence rate of the proposed algorithm. For simplicity, we do not further explore these issues and options and will consider them in future work.

\section{Initial Design}

There are many possible choices of the initial guesses $\boldsymbol{h}_{0}$, provided that they are feasible to the original non-convex problem. In other words, $\boldsymbol{h}_{0}$ should at least satisfy all constraints, while the actual approximation error in the objective function is less critical. We propose to find such initial guess by defining a virtual phase response for a given magnitude response. This allows us to relax the original non-convex problem to a convex problem so that the constraints can be easily specified under the convex programming framework described in (3-2). Using this result, we can easily find an appropriate $\boldsymbol{h}_{0}$, even though some of the 
constraints, if not all, are in general non-convex constraints as in (3-7).

As observed in the uniform linear array and other symmetric arrays with isotropic elements, the desired phase response of the main beam is always assumed to be zero. In this paper, this idea is extended to general array geometry and the virtual phase response is chosen as the average argument (phase) of all the elements

$$
\tilde{p}_{\Psi}(\theta, \phi)=\frac{1}{N} \sum_{n=1}^{N} \arg \left\{e^{j k_{0} \boldsymbol{p}_{n}^{T} \hat{\boldsymbol{u}}_{R}} q_{n, \Psi}(\theta, \phi)\right\}
$$

where $\arg \{\bullet\}$ denotes the argument of a complex number inside the bracket. This choice is quite natural in the case of the uniform linear array (as well as other symmetry arrays) with isotropic elements because it gives $\tilde{p}_{\Psi}(\theta, \phi)=0$.

Now consider again the problem in (3-7). The non-convex constraint in (3-7b) can be rewritten as:

$$
|| E_{\Psi}(\theta, \phi)\left|-\tilde{M}_{\Psi}(\theta, \phi)\right| \leq e_{\Psi}(\theta, \phi)
$$

where $\tilde{M}_{\Psi}(\theta, \phi)$ and $e_{\Psi}(\theta, \phi)$ are respectively defined as

$$
\tilde{M}_{\Psi}(\theta, \phi)=\frac{\left[U_{\Psi}(\theta, \phi)+L_{\Psi}(\theta, \phi)\right]}{2}
$$

and

$$
e_{\Psi}(\theta, \phi)=\frac{\left[U_{\Psi}(\theta, \phi)-L_{\Psi}(\theta, \phi)\right]}{2} .
$$

With the virtual phase response in (3-17), the constraint in (3-18) can be approximated by the following convex constraint

$$
\left|E_{\Psi}(\theta, \phi)-\tilde{M}_{\Psi}(\theta, \phi) e^{j \tilde{p}_{\Psi}(\theta, \phi)}\right| \leq e_{\Psi}(\theta, \phi), \quad(\theta, \phi) \in \Omega_{\Psi, 2} .
$$

This constraint is particularly suitable for the case when the magnitude constraint is specified in decibel scale, which is frequently encountered in power pattern synthesis problem. For example, suppose that $\tilde{M}_{\Psi}(\theta, \phi)$ corresponds to a cosecant beam pattern (in $\mathrm{dB}$ ), and $e_{\Psi}(\theta, \phi)$ specifies the maximum error (in dB) of deviation from $\tilde{M}_{\Psi}(\theta, \phi)$. Then, solving the problem with the constraint in (3-21) results in a beam pattern $\left|E_{\Psi}(\theta, \phi)\right|$, which has a specified error of deviation from $\tilde{M}_{\Psi}(\theta, \phi)$ in decibel scale.

Whereas for the other non-convex lower bound constraint $\left|E_{\Psi}(\theta, \phi)\right|^{2} \geq V_{\Psi}^{2}(\theta, \phi)$ in (3-7c), the strategy considered in [4] is employed. To ensure that this nonconvex constraint is satisfied, we impose a set of linear equality constraints to fix $\left|E_{\Psi}(\theta, \phi)\right|$ to values slightly larger than $V_{\Psi}(\theta, \phi)$ at several sample points of $(\theta, \phi)$. More precisely, the lower bound constraint can be approximated using a number of linear equality constraints as

$$
E_{\Psi}\left(\theta_{k}, \phi_{k}\right)=\left[V_{\Psi}\left(\theta_{k}, \phi_{k}\right)+\xi\right] e^{j \tilde{p}_{\Psi}\left(\theta_{k}, \phi_{k}\right)}
$$

$\left(\theta_{k}, \phi_{k}\right) \subset \Omega_{\Psi, 3}$, for $k=1,2 \ldots, K$, where $\xi$ is a small positive constant and $K$ is a positive integer. Note it is unnecessary to impose too many equality constraints because our primary objective is merely to ensure (3-7c) is satisfied. Using the above results, the initial guess for the problem of (3-7) can be obtained by solving the following convex problem

$$
\min _{\boldsymbol{h}_{0}} \max _{(\theta, \phi) \in \Omega_{\Psi, 1}}\left|E_{\Psi}(\theta, \phi)-M_{\Psi}(\theta, \phi) e^{j \tilde{p}_{\Psi}(\theta, \phi)}\right|
$$

subject to the constraints in (3-21) and (3-22). The initial guess found in this way is reasonable because the original non-convex problem in (3-7) is similar to a minimax problem after linearization. Alternatively, it is possible to use LS objective function instead of the minimax objective function in (3-23). However, computer simulations suggest that the LS initial guess generally leads to a slightly slower convergence although the resulting beam pattern is nearly identical to that obtained using the abovementioned initial guess. For simplicity, we shall stick with the initial guess obtained by solving (3-23).

\section{E. Design Procedure}

Without loss of generality, we particularly describe the general design procedure for the power pattern synthesis problem considered in (3-7). It is easy to modify the procedure for a variety of pattern synthesis problems with more complicated array settings, as we will illustrate in Section IV. Given number of elements $N$, array geometry, element pattern function $f$, and desired power pattern, the procedure can be summarized as follows:

Step 1: Define the virtual phase response $\tilde{p}_{\Psi}(\theta, \phi)$ in the non-sidelobe region according to (3-17), derive the convex constraints from the original non-convex constraints according to (3-18) - (3-22), and formulate and solve the resulting convex optimization problem in (3-23) for an initial guess $\boldsymbol{h}_{0}$.

Step 2: Set iteration number $k=1$, linearize the power pattern function $H_{\Psi}\left(\boldsymbol{h}_{k}+\boldsymbol{\delta}\right)$ according to (3-13), define the maximum norm bound $\delta_{\max }$, and formulate and solve the convex optimization problem in (3-16) for a linear update vector $\delta$.

Step 3: Update the solution $\boldsymbol{h}_{k+1}=\boldsymbol{h}_{k}+\boldsymbol{\delta}$ and increase $k$ by one. Repeat from Step 2 until the relative change of two successive solutions is insignificant or the maximum number of iteration is reached.

\section{DESIGN EXAMPLES}

In the following examples, the maximum norm of the linear update vector $\delta$ is chosen as $\delta_{\max }=1 \times 10^{-3} . \theta$ and $\phi$ are respectively discretized into $N_{\Theta}=901$ and $N_{\Phi}=1801$ equally spaced samples for an increment of $0.2^{\circ}$ per sample over the whole range of interest. All the SOCP optimization problems were solved by the CVX Matlab Toolbox [20] on a Pentium 4 personal computer.

\section{A. Example 1: Uniform Linear Array}

In this example, the power pattern synthesis of a uniform linear array with isotropic elements is considered. Since the ap- 


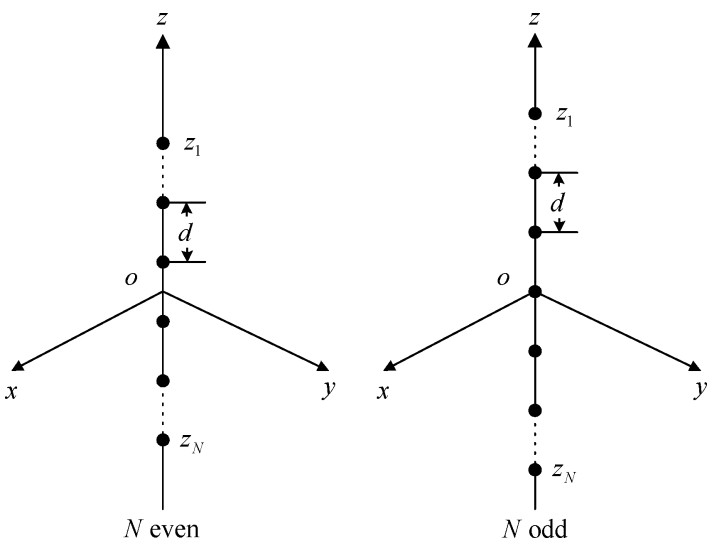

Fig. 2. Uniform linear array geometry.

proach in [7] is capable of giving an optimal pattern in this simple case, a comparison with this approach will be considered below. Such optimal result provides a gold standard to assess the performance of the proposed approach in this particular problem.

Consider the uniform linear array of $N$ elements shown in Fig. 2, where the array lies on the $z$-axis and has a center of symmetry at $z=0$. Let $d$ be the distance between two adjacent elements. The position vector of the $n$th element is then given by $\boldsymbol{p}_{n}=\left[0,0, z_{n}\right]^{T} ; z_{n}=d[(N-1) / 2-(n-1)]$ and hence we have $\boldsymbol{p}_{n}^{T} \hat{\boldsymbol{u}}_{R}=z_{n} \cos \theta$ for $n=1, \ldots, N$. Since coordinate transformation is not required for linear array geometry and $q_{n, \Psi}(\theta, \phi)=1$ for isotropic elements, the radiation pattern of the uniform linear array is simply given by

$$
E_{\Psi}(\theta)=\sum_{n=1}^{N} a_{n} e^{j k_{0} z_{n} \cos \theta}
$$

according to (2-9). Also, it is independent of $\phi$-direction.

As an illustration, suppose that we want to synthesize a beam pattern having minimum sidelobe level for $\theta \in \Omega_{\Psi}^{S L}$ and a cosecant squared main beam $C_{\mathrm{dB}}(\theta)$ (in decibel scale) with maximum allowable error $\pm \sigma \mathrm{dB}$ for $\theta \in \Omega_{\Psi}^{M B}$, together with a prescribed upper bound magnitude constraint for $\theta \in \Omega_{\Psi}^{U B}$. The problem can be formulated as

$$
\begin{aligned}
& \min _{a_{n}, \varepsilon} \varepsilon \\
& \text { subject to }\left|E_{\Psi}(\theta)\right|^{2} \leq \varepsilon, \quad \theta \in \Omega_{\Psi}^{S L} \\
& L_{\Psi}^{2}(\theta) \leq\left|E_{\Psi}(\theta)\right|^{2} \leq U_{\Psi}^{2}(\theta), \quad \theta \in \Omega_{\Psi}^{M B} \\
& \qquad\left|E_{\Psi}(\theta)\right|^{2} \leq \mu, \quad \theta \in \Omega_{\Psi}^{U B}
\end{aligned}
$$

where $L_{\Psi}(\theta)=10^{\left[C_{\mathrm{dB}}(\theta)-\sigma\right] / 20}$ and $U_{\Psi}(\theta)=10^{\left[C_{\mathrm{dB}}(\theta)+\sigma\right] / 20}$. The array pattern with the following parameters: $N=31 ; d=$ $0.5 \lambda ; \sigma=0.2 ; \Omega_{\Psi}^{M B} \in\left[95^{\circ}, 120^{\circ}\right] ; \Omega_{\Psi}^{U B} \in\left[90^{\circ}, 95^{\circ}\right] ; \mu=1$; and $\Omega_{\Psi}^{S L} \in\left[0,90^{\circ}\right] \cap\left[125^{\circ}, 180^{\circ}\right]$ will be considered below.

For comparison purpose, we use the method in [7] (Matlab code is available in [20]) to solve the problem in (4-2). Its basic idea is to reformulate (4-2) with respect to the autocorrelation of $a_{n}$, so that $\left|E_{\Psi}(\theta)\right|^{2}$ becomes a linear function of the autocorrelation coefficients. Hence, the equivalent linear programming problem can be solved optimally. For practical implementation, an additional step of spectral factorization is required to extract $a_{n}$ from the optimal autocorrelation coefficients. Fig. 3(a) and (b) show respectively the patterns obtained after convex optimization and spectral factorization. We notice a slight difference between these two patterns because of the numerical error caused by spectral factorization, especially when $N$ is large. That is, the factorized result may not exactly assemble the optimal autocorrelation coefficients in the reverse process. Nevertheless, we only focus on the optimal pattern derived from the autocorrelation coefficients.

From the discussions in Section III-C, an initial guess of the proposed algorithm is first obtained by solving the following convex problem:

$$
\begin{aligned}
\min _{a_{n}, \tilde{\varepsilon}} \tilde{\varepsilon} & \\
\text { subject to }\left|E_{\Psi}(\theta)\right| & \leq \tilde{\varepsilon}, \quad \theta \in \Omega_{\Psi}^{S L} \\
\left|E_{\Psi}(\theta)-\tilde{M}_{\Psi}(\theta)\right| & \leq e_{\Psi}(\theta), \quad \theta \in \Omega_{\Psi}^{M B} \\
\left|E_{\Psi}(\theta)\right| & \leq \mu^{0.5}, \quad \theta \in \Omega_{\Psi}^{U B}
\end{aligned}
$$

where $\tilde{M}_{\Psi}(\theta)$ and $e_{\Psi}(\theta)$ are defined according to (3-19) and (3-20), respectively. Here, $\tilde{\varepsilon}^{2}$ corresponds to $\varepsilon$ in the original problem in (4-2). Note, in the second constraint, the corresponding virtual phase response $\tilde{p}_{\Psi}(\theta)$ is equal to zero as defined in (3-17). Fig. 3(c) and (d) show respectively the initial and proposed array patterns. We can see that the proposed pattern have a much better sidelobe attenuation than the initial pattern, because the problem in (4-3) ignores the phase information and hence more degree of design freedom is available. Also, the proposed pattern converges to a solution which is very close to the optimal pattern shown in Fig. 3(a), even though the initial guess is far away from the optimal solution. This suggests the proposed iterative method is effective in approaching the optimal solution.

Regarding the design complexity of the proposed method, each iteration is solved in about 5 seconds, thanks to the efficient SOCP solver. The number of iterations and the design time may increase with the numbers of variables and constraints involved, but the design complexity is still affordable for nowadays personal computers.

Next, we shall demonstrate the effectiveness of the proposed approach by considering a more difficult power pattern synthesis problem of conformal arrays with arbitrary array geometries and polarization characteristics, where no optimal solution is guaranteed in general.

\section{B. Example 2: Hemi-Spherical Array}

In this example, the power pattern synthesis of a hemi-spherical array with circular polarization is considered. As mentioned earlier, the convex programming methods in [7] and [8] are not directly applicable to such nonlinear array geometry and directive elements.

For simulation purpose, we follow the common practice used in related studies and assume that the radiation characteristics of each element are described by certain established empirical models. The use of such model not only helps us to verify the 


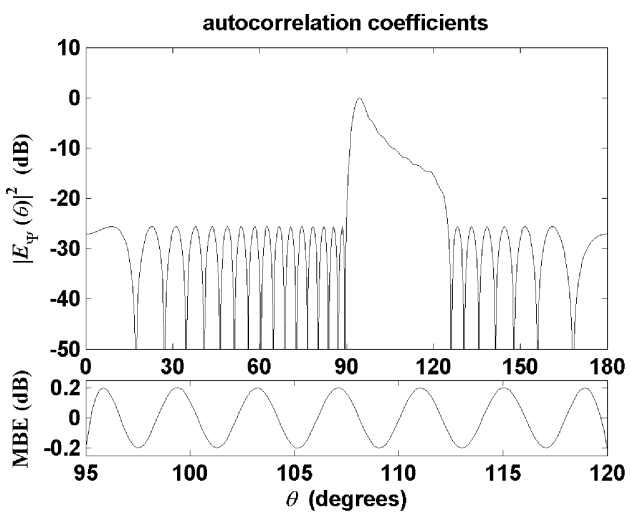

(a)

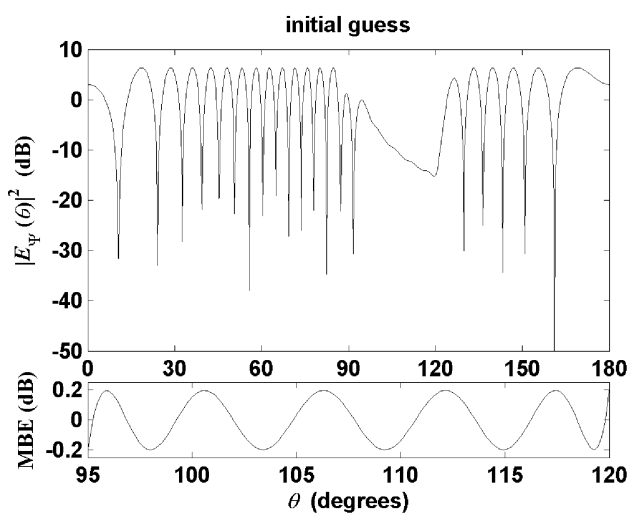

(c)

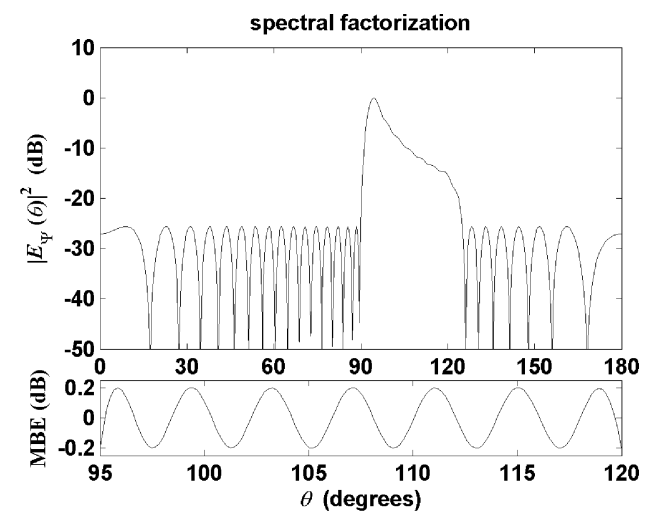

(b)

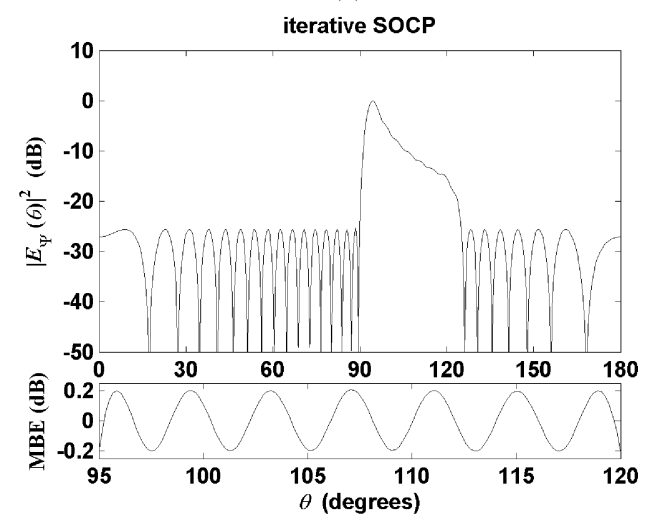

(d)

Fig. 3. Synthesized power patterns obtained using (a) optimal autocorrelation coefficients, (b) complex excitations factorized from autocorrelation coefficients, (c) initial complex excitations, and (d) proposed complex excitations. MBE: Main Beam Error.

usefulness of the proposed approach more conveniently, but also allows the antenna designers to predict the radiation pattern of the target array and determine the required configurations, such as the number of antennas used and their locations, to meet a given specification before proceeding to construct the real antenna system. Once the real system has been constructed, it is also possible to use the measured element radiation patterns in the problem formulation so as to perform final tuning of the resultant beam pattern.

As an illustration, the individual element pattern is assumed to be the lowest order circular patch model used in [13] as follows

$$
\begin{aligned}
& f_{\Theta_{n}^{\prime}}\left(\theta_{n}^{\prime}, \phi_{n}^{\prime}\right)= \begin{cases}e^{j \phi_{n}^{\prime}} A_{\Theta}\left(\theta_{n}^{\prime}\right) & \theta_{n}^{\prime} \in\left[0,90^{\circ}\right] \\
0 & \text { otherwise }\end{cases} \\
& f_{\Phi_{n}^{\prime}}\left(\theta_{n}^{\prime}, \phi_{n}^{\prime}\right)= \begin{cases}-j e^{j \phi_{n}^{\prime}}\left(\cos \theta_{n}^{\prime}\right) A_{\Phi}\left(\theta_{n}^{\prime}\right) & \theta_{n}^{\prime} \in\left[0,90^{\circ}\right] \\
0 & \text { otherwise }\end{cases}
\end{aligned}
$$

where $A_{\Theta}\left(\theta_{n}^{\prime}\right)=J_{2}\left(0.55 \pi \sin \theta_{n}^{\prime}\right)-J_{0}\left(0.55 \pi \sin \theta_{n}^{\prime}\right)$ and $A_{\Phi}\left(\theta_{n}^{\prime}\right)=J_{2}\left(0.55 \pi \sin \theta_{n}^{\prime}\right)+J_{0}\left(0.55 \pi \sin \theta_{n}^{\prime}\right)$. Following [21], an isosahedron type of element distribution is used in the hemi-spherical array and it is given by the following parameters:

$$
\alpha_{p}=p 15^{\circ}, p=0,1,2,3
$$

$$
\beta_{p q}= \begin{cases}0 & p=0 \text { and } q=0 \\ \left(\frac{q}{p}\right) 72^{\circ} & p=1,2,3, \text { and } q=0, \ldots, 5 p-1 .\end{cases}
$$

In this distribution, the average element spacing is approximately given by $0.3 R_{0}$, where $R_{0}$ is the radius of the sphere ( $R_{0}=\lambda$ in this example). Fig. 4 shows the corresponding array geometry, in which the polar coordinate of each element is $\left(R_{0}, \alpha_{p}, \beta_{p q}\right)$ and there are $N=31$ elements. All the elements are assumed to be pointed towards the radial direction. For coordinate transformation mentioned in Section II, the required rotation matrix of the element located at $\left(R_{0}, \alpha_{p}, \beta_{p q}\right)$ is given by

$\boldsymbol{M}_{p q}=\left[\begin{array}{ccc}\cos \alpha_{p} & 0 & -\sin \alpha_{p} \\ 0 & 1 & 0 \\ \sin \alpha_{p} & 0 & \cos \alpha_{p}\end{array}\right]\left[\begin{array}{ccc}\cos \beta_{p q} & \sin \beta_{p q} & 0 \\ -\sin \beta_{p q} & \cos \beta_{p q} & 0 \\ 0 & 0 & 1\end{array}\right]$.

After simple re-indexing to replace $p$ and $q$ by $n$, the polarization components of the array in the elevation and azimuth directions can be respectively expressed in term of $a_{n}$ according to (2-6) and (2-7).

Now suppose that we want to synthesize a beam pattern having the following desired properties: 


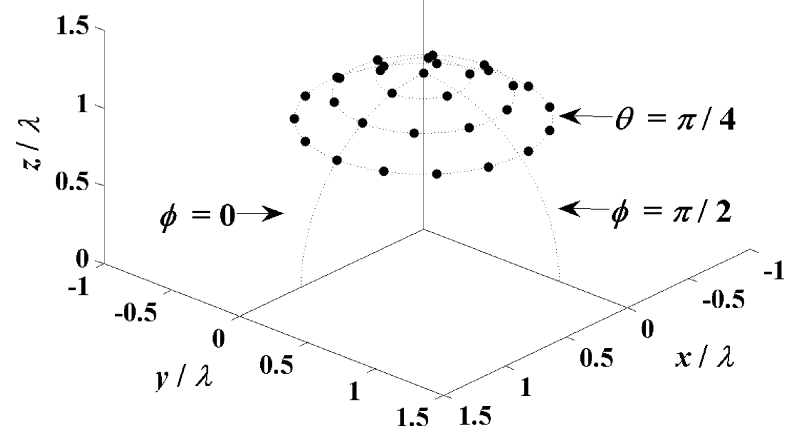

Fig. 4. Hemi-spherical array geometry with radius $R_{0}=\lambda$.

i) Desired co-polarization component $\left|E_{\Theta}(\theta, \phi)\right|^{2}$ :

$$
\begin{cases}\in[-0.1 \mathrm{~dB}, 0.1 \mathrm{~dB}] & \theta \in\left[-5^{\circ}, 5^{\circ}\right] \text { and } \phi \in\left[0^{\circ}, 180^{\circ}\right] \\ \leq-15 \mathrm{~dB} & \theta \in\left[-30^{\circ},-20^{\circ}\right] \text { and } \phi \in\left[0^{\circ}, 180^{\circ}\right] \\ \text { to be minimized } & |\theta| \in\left[20^{\circ}, 90^{\circ}\right] \text { and } \phi \in\left[0^{\circ}, 180^{\circ}\right] .\end{cases}
$$

ii) Desired cross-polarization component $\left|E_{\Phi}(\theta, \phi)\right|^{2}$ : to be minimized, $\theta \in\left[-90^{\circ}, 90^{\circ}\right]$ and $\phi \in\left[0^{\circ}, 180^{\circ}\right]$.

In other words, the problem objective is to minimize the sidelobes and cross-polarization simultaneously. Also, the beam pattern is shaped to have a flat magnitude response for $\theta \in\left[-5^{\circ}, 5^{\circ}\right]$ and $\phi \in\left[0^{\circ}, 180^{\circ}\right]$, and a prescribed sidelobe attenuation of $-15 \mathrm{~dB}$ for $\theta \in\left[-30^{\circ},-20^{\circ}\right]$ and $\phi \in\left[0^{\circ}, 180^{\circ}\right]$. Note that we only consider the space for $z \geq 0$ (i.e., $\theta \in\left[-90^{\circ}, 90^{\circ}\right]$ and $\phi \in\left[0^{\circ}, 180^{\circ}\right]$ ), because the radiation field at lower half of the sphere is negligible according to the element pattern function and array geometry we respectively defined in (4-4) and (4-5). Equivalently, the problem can be posed as:

$$
\begin{gathered}
\min _{a_{n}, \varepsilon} \varepsilon \\
\text { subject to } 10^{-0.1 / 10} \\
\leq\left|E_{\Theta}(\theta, \phi)\right|^{2} \leq 10^{0.1 / 10}, \theta \in\left[-5^{\circ}, 5^{\circ}\right] \cap \phi \in\left[0^{\circ}, 180^{\circ}\right] \\
\left|E_{\Theta}(\theta, \phi)\right|^{2} \leq 10^{-15 / 10}, \theta \in\left[-30^{\circ},-20^{\circ}\right] \cap \phi \in\left[0^{\circ}, 180^{\circ}\right] \\
\left|E_{\Theta}(\theta, \phi)\right|^{2} \leq \varepsilon, \quad|\theta| \in\left[20^{\circ}, 90^{\circ}\right] \cap \phi \in\left[0^{\circ}, 180^{\circ}\right] \\
\left|E_{\Phi}(\theta, \phi)\right|^{2} \leq \varepsilon, \quad \theta \in\left[-90^{\circ}, 90^{\circ}\right] \cap \phi \in\left[0^{\circ}, 180^{\circ}\right] .
\end{gathered}
$$

The corresponding convex problem for determining the initial guess can be written as:

$$
\begin{aligned}
& \min _{a_{n}, \tilde{\varepsilon}} \tilde{\varepsilon} \\
& \text { subject to }\left|E_{\Theta}(\theta, \phi)-\tilde{M}_{\Theta}(\theta, \phi) e^{j \tilde{p}_{\Theta}(\theta, \phi)}\right| \\
& \quad \leq e_{\Theta}\left(\theta, \phi_{T}\right), \theta \in\left[-5^{\circ}, 5^{\circ}\right] \cap \phi \in\left[0^{\circ}, 180^{\circ}\right] \\
&\left|E_{\Theta}(\theta, \phi)\right| \leq 10^{-15 / 20}, \theta \in\left[-30^{\circ},-20^{\circ}\right] \cap \phi \in\left[0^{\circ}, 180^{\circ}\right] \\
&\left|E_{\Theta}(\theta, \phi)\right| \leq \tilde{\varepsilon},|\theta| \in\left[20^{\circ}, 90^{\circ}\right] \cap \phi \in\left[0^{\circ}, 180^{\circ}\right] \\
&\left|E_{\Phi}(\theta, \phi)\right| \leq \tilde{\varepsilon}, \quad \theta \in\left[-90^{\circ}, 90^{\circ}\right] \cap \phi \in\left[0^{\circ}, 180^{\circ}\right]
\end{aligned}
$$

where $\tilde{p}_{\Theta}(\theta, \phi), \tilde{M}_{\Theta}(\theta, \phi)$ and $e_{\Theta}(\theta, \phi)$ are determined according to (3-17), (3-19) and (3-20), respectively. Fig. 5 compares the results of the initial and proposed co- and cross-polarization patterns. It can be seen that the proposed patterns satisfy all the required constraints, while achieving much lower sidelobes and cross-polarization than the initial pattern. This illustrates the design flexibility and freedom of the proposed approach in handling non-convex magnitude constraints in general power pattern synthesis problems.

\section{Example 3: Circular-Arc Array With Mutual Coupling}

It has been demonstrated that the radiation pattern is significantly degraded when mutual coupling is not considered [22]. In this example, we extend the proposed pattern synthesis method to include the effect of mutual coupling. To this end, we first rewrite (3-9) as:

$$
E_{\Psi}=(\boldsymbol{C a})^{T} \boldsymbol{q}_{\Psi}
$$

where the interaction among the elements are characterized by a $(N \times N)$ complex matrix $\boldsymbol{C}$ called mutual coupling matrix. In general, the coefficients of $\boldsymbol{C}$ are difficult to determine analytically, but can be calculated using numerical techniques suggested in [1]. Consequently, the proposed method can be applied to handle the mutual coupling effect if $\boldsymbol{q}_{\Psi}$ in (3-9) is replaced by $\boldsymbol{C}^{T} \boldsymbol{q}_{\Psi}$ and subsequent steps described in Section III are derived similarly.

As a comparison, we focus on the same array settings and specifications studied in [22], wherein an 18-element circular-arc array with radius $R_{0}=4.3 \lambda$ is considered. The array is placed on the $x-y$ plane and only the horizontal plane (i.e., the elevation angle of $\theta=90^{\circ}$ ) is considered. The spacing of adjacent elements is $0.53 \lambda$. The azimuth location of the $n$th element is $\beta_{n}=\Delta \beta(N-2 n+2) / 2, n=1, \ldots, N$ and $\Delta \beta$ is the angular separation. Each element has a cosine isolated pattern, which is given by $f_{\Psi_{n}^{\prime}}\left(\phi_{n}^{\prime}\right)=\max \left\{\cos \phi_{n}^{\prime}, 0\right\}$. To calculate the total radiation pattern, each element undergoes a coordinate transformation with the rotation matrix

$$
M_{n}=\left[\begin{array}{ccc}
\cos \beta_{n} & \sin \beta_{n} & 0 \\
-\sin \beta_{n} & \cos \beta_{n} & 0 \\
0 & 0 & 1
\end{array}\right] .
$$

A cosecant main beam with unequal sidelobe level is synthesized according to the pre-defined pattern masks shown as dashed lines in Fig. 6(a). Using the notations defined previously, the pattern masks can be translated into the following specifications

$$
\left|E_{\Psi}(\phi)\right|^{2}: \begin{cases}\leq-20 \mathrm{~dB} & \phi \in\left[-180^{\circ}, 0^{\circ}\right] \\ \in[-2 \mathrm{~dB}, 0 \mathrm{~dB}] & \phi \in\left[5^{\circ}, 10^{\circ}\right] \\ \in\left[C_{\mathrm{dB}}(\phi)-2 \mathrm{~dB}, C_{\mathrm{dB}}(\phi)\right] & \phi \in\left[10^{\circ}, 35^{\circ}\right] \\ \leq-30 \mathrm{~dB} & \phi \in\left[40^{\circ}, 180^{\circ}\right]\end{cases}
$$

where $C_{\mathrm{dB}}(\phi)$ denotes the cosecant squared function in decibel scale.

In [22], the alternating projection method is used to find a pattern lying within the required pattern masks. The resulting 


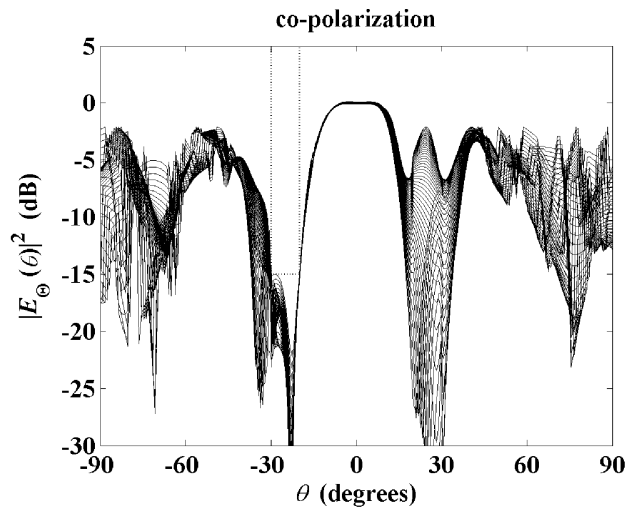

(a)

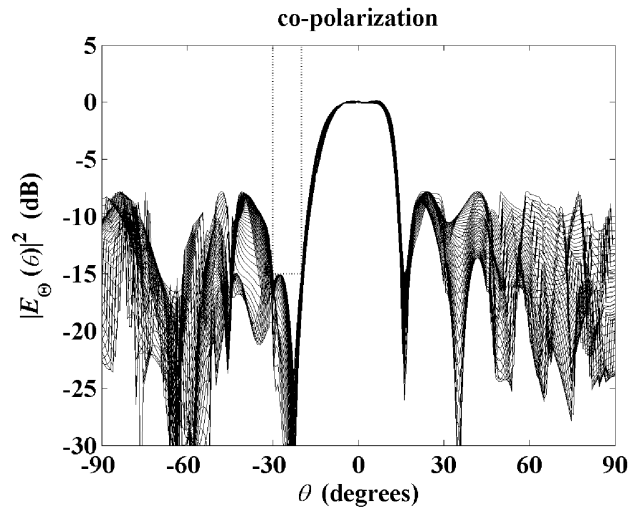

(c)

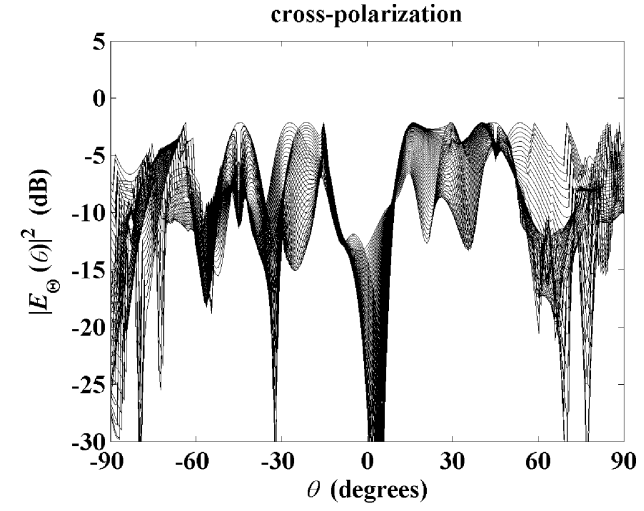

(b)

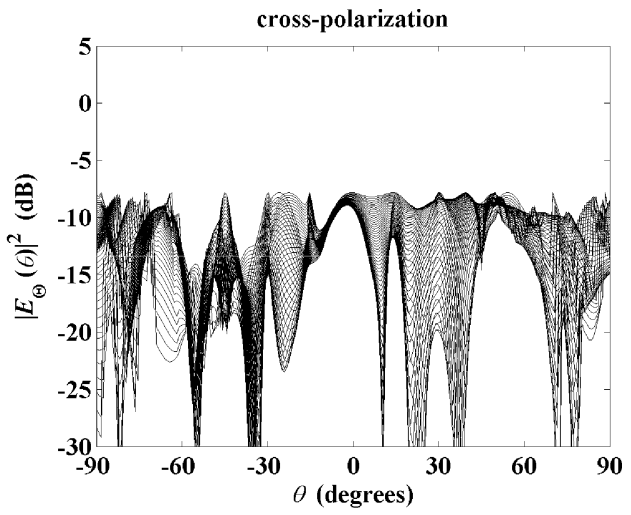

(d)

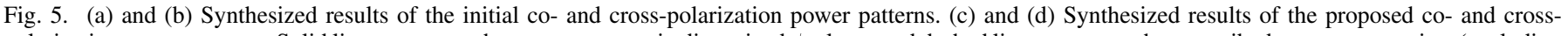

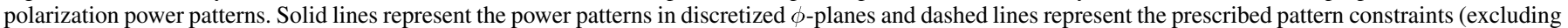
the main beam constraint for clarity).

pattern shown in Fig. 6(a) almost fulfills the requirements, except for a portion of the pattern near the band edge. In particular, the worst case errors deviated from the desired specifications in (4-11) are $0.545 \mathrm{~dB}\left(\phi=0^{\circ}\right), 0.049 \mathrm{~dB}\left(\phi=5^{\circ}\right), 0.247$ $\mathrm{dB}\left(\phi=35^{\circ}\right)$ and $1.776 \mathrm{~dB}\left(\phi=40^{\circ}\right)$, respectively. On the other hand, the proposed method is employed to minimize the sidelobe level at $\phi \in\left[40^{\circ}, 180^{\circ}\right]$, while the other pattern requirements are left as constraints to the minimization problem. From Fig. 6(b), the sidelobe level of the proposed pattern at $\phi \in\left[40^{\circ}, 180^{\circ}\right]$ is found to be $-29.6 \mathrm{~dB}$, while the prescribed specifications in other three regions of $\phi$ are precisely satisfied. Comparing with the pattern obtained using the alternating projection method, the proposed pattern has slightly better performance in every target regions of $\phi$ defined in (4-11). The main reason is that the alternating projection method aims at finding a suitable pattern that closely matches with the pattern masks, rather than optimizing the pattern according to the masks, as suggested in a comprehensive work for this method [23]. Therefore, the resulting pattern is in general not guaranteed to satisfy all the requirements, especially when the masks are not chosen properly. However, it is not always straightforward to find such masks because we do not have prior knowledge on the ultimate performance of the array at hand. For example, if the required sidelobe level at $\phi \in\left[-180^{\circ}, 0^{\circ}\right]$ is increased to $-15 \mathrm{~dB}$, one has to define the masks at $\phi \in\left[40^{\circ}, 180^{\circ}\right]$ in a trial and error manner. For the proposed method, such intervention is not re- quired. Fig. 7(a) shows another optimized power pattern obtained by setting the maximum sidelobe level at $\phi \in\left[-180^{\circ}, 0^{\circ}\right]$ to $-15 \mathrm{~dB}$. We can see that the sidelobe level at $\phi \in\left[40^{\circ}, 180^{\circ}\right]$ is now minimized to about $-32.7 \mathrm{~dB}$. Of course, if this minimal value is employed as the upper mask at $\phi \in\left[40^{\circ}, 180^{\circ}\right]$ in the alternating projection method, a reasonably good pattern that almost satisfies the masks can still be obtained. Nevertheless, the proposed method is able to provide more accurate results than the alternating projection method in most cases.

We now illustrate the effect of mutual coupling by repeating the synthesis problem without taking the mutual coupling into account for the desired pattern where the maximum sidelobe level is set to $-15 \mathrm{~dB}$ at $\phi \in\left[-180^{\circ}, 0^{\circ}\right]$. The degradation of the resulting pattern due to mutual coupling is demonstrated in Fig. 7(b). We can see that it significantly deviates from the desired specifications as the mutual coupling is not compensated. This suggests that the proposed method provides an attractive alternative to conventional methods for solving the general power pattern synthesis problem of conformal arrays with the effect of the mutual coupling. Table I summarizes the dynamic range ratios for all array excitations designed in Examples 1, 2 and 3. Those obtained using the proposed method are all reasonable.

\section{CONCLusions}

An iterative SOCP method for the power pattern synthesis of narrowband conformal arrays is presented. The non-convex 


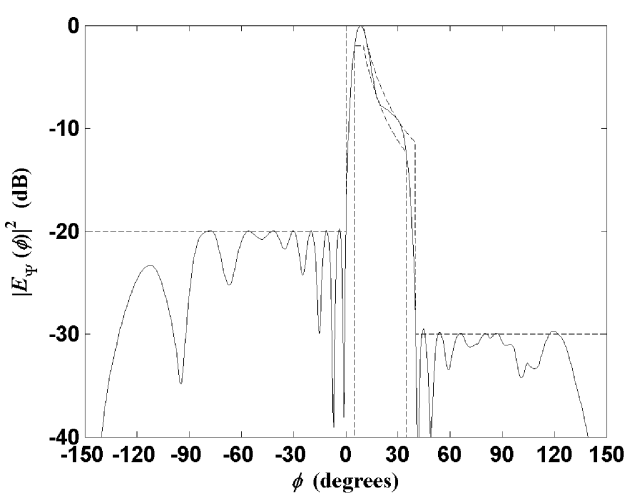

(a)

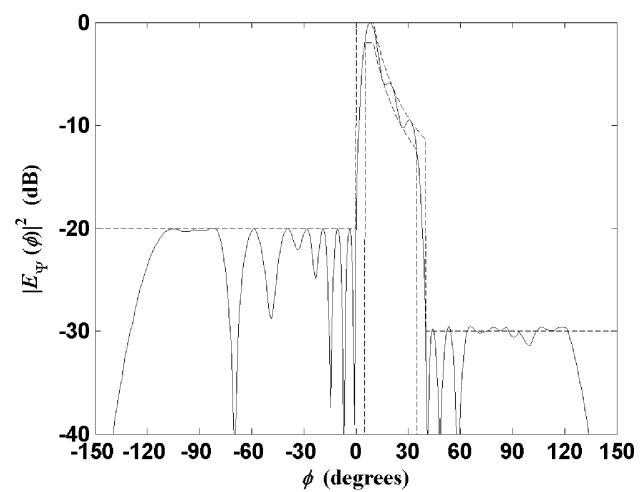

(b)

Fig. 6. Synthesized power patterns including mutual coupling effect, obtained using (a) the alternating projection method, and (b) the proposed method. Dashed lines show the required pattern masks.

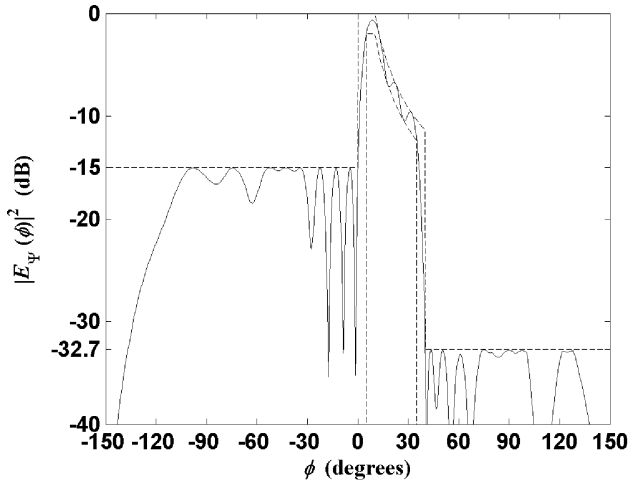

(a)

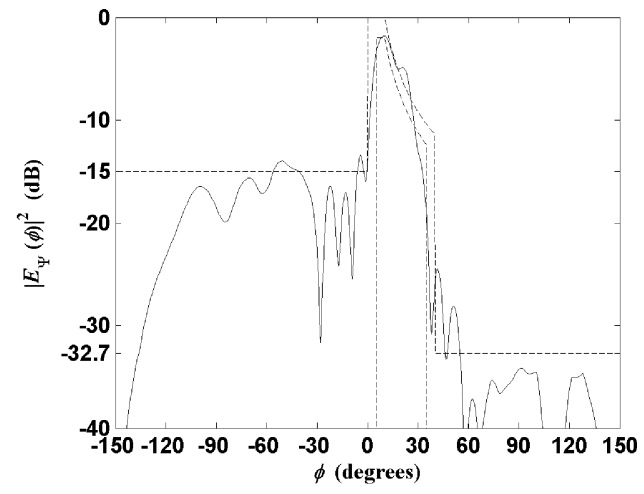

(b)

Fig. 7. (a) Synthesized power pattern including mutual coupling effect. (b) Synthesized power pattern excluding mutual coupling effect. Dashed lines show the required pattern masks.

TABLE I

DYNAMIC RANGE RATIOS IN EXAMPLES 1, 2 AND 3.

\begin{tabular}{l||c}
\hline \hline & Dynamic Range Ratio \\
\hline \hline Example 1 & \\
\hline Spectral factorization (Fig. 3b) & 9.1989 \\
\hline Initial guess (Fig. 3c) & 35.9446 \\
\hline Proposed method (Fig. 3d) & 7.7371 \\
\hline \hline Example 2 & \\
\hline Initial guess (Fig. 5a and 5b) & 171.0002 \\
\hline Proposed method (Fig. 5c and 5d) & 28.4675 \\
\hline \hline Example 3 & \\
\hline Alternating projection (Fig. 6a) & 19.8979 \\
\hline Proposed method (Fig. 6b) & 7.5617 \\
\hline Proposed method (Fig. 6c) & 5.8526 \\
\hline Proposed method (Fig. 6d) & 6.3996 \\
\hline \hline
\end{tabular}

power pattern synthesis problem is solved via a sequence of linear approximations in which each subproblem is solved using SOCP. Design results show that the proposed method is an attractive alternative to traditional design methods in tackling a wide range of pattern synthesis problems with various types of magnitude constraints, because of its generality and flexibility. In particular, the proposed method is able to design a power pattern that is close to the optimal one in the case of uniform linear array with isotropic elements, and is also applicable to more complicated power pattern synthesis problems involving arbitrary array geometries and polarization characteristics, and mutual coupling effect.

The proposed method can also be extended to handle the model errors, say due to inexact radiation characteristics and location of antenna elements. By treating the unknown errors as random variables with appropriate bounds specified by the antenna designers, the corresponding uncertainties can be taken into account in the design procedure to reduce the sensitivity of the designed pattern at the expense of slightly degraded performance. This is usually referred to as the robust beamformer design problem. Due to page limitation, we will report this interesting problem in future work.

\section{ACKNOWLEDGMENT}

The authors would like to thank the anonymous reviewers for their useful comments and suggestions, which greatly improved the quality of this paper.

\section{REFERENCES}

[1] L. Josefsson and P. Persson, Conformal Array Antenna Theory and Design. Piscataway-Hoboken, NJ: IEEE Press-Wiley, 2006.

[2] Y. Liu and Z. Lin, "On the applications of the frequency-response masking technique in array beamforming," Circ., Syst. Signal Processing, Special Issue on Computationally Efficient Digital Filters: Design Techniques and applications, vol. 25, no. 2, pp. 201-224, 2006. 
[3] K. F. C. Yiu, X. Yang, S. Nordholm, and K. L. Teo, "Near-field broadband beamformer design via multidimensional semi-infinite-linear programming technique," IEEE Trans. Speech Audio Process., vol. 11, no. 6, pp. 725-732, Nov. 2003.

[4] H. Lebret and S. Boyd, "Antenna array pattern synthesis via convex optimization," IEEE Trans. Signal Processing, vol. 45, pp. 526-532, Mar. 1997.

[5] D. P. Scholnik and J. O. Coleman, "Formulating wideband array-pattern optimization," in Proc. IEEE Int. Conf. Phased Array Syst. Technol., May 2000, pp. 489-492.

[6] J. O. Coleman, D. P. Scholnik, and P. E. Cahill, "Synthesis of a polarization-controlled pattern for a wideband array by solving a secondorder cone program," in Proc. IEEE Int. Symp. Antennas and Propagation Society, Jul. 2005, vol. 28, pp. 437-440.

[7] S. P. Wu, S. Boyd, and L. Vandenberghe, "FIR filter design via spectral factorization and convex optimization," in Applied and Computational Control, Signals and Circuits, B. Datta, Ed. New York: Birkhauser, 1998, vol. 1, pp. 215-245.

[8] T. Davidson, T. Luo, and J. Sturm, "Linear matrix inequality formulation of spectral mask constraints with applications to FIR filter design," IEEE Trans. Signal Processing, vol. 50, pp. 2702-2715, 2002.

[9] F. J. A. Pena, J. A. Rodriguez-Gonzalez, E. Villanueva-Lopez, and S. R. Rengarajan, "Genetic algorithms in the design and implementation of antenna array patterns," IEEE Trans. Antennas Propag., vol. 47, no. 3, pp. 506-510, Mar. 1999.

[10] D. W. Boeringer and D. H. Werner, "Particle swarm optimization versus genetic algorithms for phased array synthesis," IEEE Trans. Antennas Propag., vol. 52, no. 3, pp. 771-779, Mar. 2004.

[11] J. A. Ferreira and F. Ares, "Pattern synthesis of conformal arrays by the simulated annealing technique," Electron. Lett., vol. 33, no. 14, pp. $1187-1189,1997$

[12] F. Wang, V. Balakrishnan, P. Y. Zhou, J. J. Chen, R. Yang, and C. Frank, "Optimal array pattern synthesis using semidefinite programming," IEEE Trans. Signal Process., vol. 51, no. 5, pp. 1172-1183, May 2003.

[13] L. I. Vaskelainen, "Iterative least-sauares synthesis methods for conformal array antennas with optimizaed polarization and frequency properties," IEEE Trans. Antennas Propag., vol. 45, no. 7, pp. 1179-1185, Jul. 1997.

[14] W. S. Lu and T. Hinamoto, "Optimal design of IIR digital filters with robust stability using cone-quadratic-programming updates," IEEE Trans. Signal Processing, vol. 51, pp. 1581-1592, Jun. 2003.

[15] W. S. Lu and T. Hinamoto, "Optimal design of IIR frequency- response-masking filters using second-order cone programming," IEEE Trans. Circuits Syst. I, vol. 50, pp. 1402-1412, Nov. 2003.

[16] J. Nocedal and S. J. Wright, Numerical Optimization. Berlin: Springer, 1999, Series in Operations Research.

[17] T. Milligan, "More applications of Euler rotation angles," IEEE Antennas Propag. Mag., vol. 41, no. 4, pp. 78-83, Aug. 1999.

[18] T. A. Milligan, Modern Antenna Array, 2nd ed. Piscataway-Hoboken, NJ: IEEE Press-Wiley, 2005.

[19] R. S. Elliott, Antenna Theory and Design. Englewood Cliffs, NJ: Prentice Hall, 1981.

[20] M. Grant, S. Boyd, and Y. Ye, "CVX Version 1.1. Matlab Software for Disciplined Convex Programming," 2007 [Online]. Available: www. stanford.edu/ boyd/cvx/

[21] D. L. Sengupta, T. M. smith, and R. W. Larson, "Radiation characteristics of a spherical array of circularly polarized elements," IEEE Trans. Antennas Propag., vol. 16, no. 1, pp. 2-7, Jan. 1968.

[22] L. Josefsson and P. Persson, "Conformal array synthesis including mutual coupling," Electron. Lett., vol. 35, no. 8, pp. 625-627, 1999.

[23] O. M. Bucci, G. D’Eila, G. Mazzarella, and G. Panariello, "Antenna pattern synthesis: A new general approach," Proc. IEEE, vol. 82, no. 3, pp. 358-371, 1994.

[24] N. Kojima, K. Hariu, and I. Chiba, "Low sidelobe pattern synthesis using projection method with mutual coupling compensation," in Proc. IEEE Int. Symp. on Phased Array Systems and Technology, 2003, pp. $559-564$.
[25] H. Steyskal, "Pattern synthesis for a conformal wing array," in Proc. IEEE Aerospace Conf., 2002, vol. 2, pp. 2-819-2-824.

[26] D. A. Wingert and B. M. Howard, "Potential impact of smart electromagnetic antennas on aircraft performance and design," in Proc. NATO Workshop on Smart Electromagnetic Antenna Structures, Brussels, Nov. 1996, pp. 1-10.

[27] M. Kanno, T. Hashimura, T. Katada, M. Sato, K. Fukutani, and A. Suzuki, "Digital beam forming for conformal active array antenna," in Proc. IEEE Int. Symp. on Phased Array Systems and Technology, Oct. 1996, pp. 37-40.

[28] S. W. Schneider, C. Bozada, R. Dettmer, and J. Tenbarge, "Enabling technologies for future structurally integrated conformal apertures," in IEEE AP-S Int. Symp. Digest, Boston, Jul. 2001, pp. 330-333.

[29] M. A. Hopkins, J. M. Tuss, A. J. Lockyer, K. Alt, R. Kinslow, and J. N. Kudva, "Smart skin conformal load-bearing antenna and other smart structures developments," in Proc. American Institute of Aeronautics and Astronautics (AIAA), Structures, Structural Dynamics and Materials Conf., 1997, vol. 1, pp. 521-530.

[30] E. Vourch, C. Caille, M. J. Martin, J. R. Mosig, A. Martin, and P. O. Iversen, "Conformal array antenna for LEO observation platforms," in IEEE AP-S Int. Symp. Digest, 1998, pp. 20-23.

[31] G. Caille, E. Vourch, M. J. Martin, J. R. Mosig, and A. Martin Polegre, "Conformal array antenna for observation platforms in low earth orbit," IEEE Antennas Propag. Mag., vol. 44, no. 3, pp. 103-104, Jun. 2002.

[32] P. Knott, "Design and experimental results of a spherical antenna array for a conformal array demonstrator," in Int. ITG Conf. Antennas - INICA, Mar. 2007, pp. 120-123.

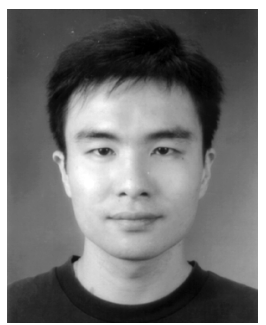

K. M. Tsui received the B.Eng., M.Phil., and Ph.D. degrees in electrical and electronic engineering from the University of Hong Kong, in 2001, 2004 and 2008 , respectively.

He is currently working as a Postdoctoral Fellow in the Department of Electrical and Electronic Engineering at the University of Hong Kong. His main research interests are in array signal processing, highspeed AD converter architecture, biomedical signal processing, digital signal processing, multirate filter bank and wavelet design, and digital filter design, re-

alization and application.

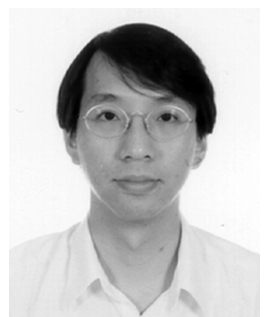

S. C. Chan (S'87-M'92) received the B.Sc. (Eng) and $\mathrm{Ph} . \mathrm{D}$. degrees from the University of Hong Kong, in 1986 and 1992, respectively.

He joined City Polytechnic of Hong Kong in 1990 as an Assistant Lecturer and later as a University Lecturer. Since 1994, he has been with the Department of Electrical and Electronic Engineering, University of Hong Kong, and is now a Professor. He was a Visiting Researcher in Microsoft Corporation, Redmond, USA, Microsoft, Beijing, China, University of Texas at Arlington and Nanyang Technological University, Singapore. His research interests include fast transform algorithms, filter design and realization, multirate and biomedical signal processing, communications and array signal processing, high-speed $\mathrm{AD}$ converter architecture, bioinformatics and image-based rendering.

Dr. Chan is currently a member of the Digital Signal Processing Technical Committee of the IEEE Circuits and Systems Society and an Associate Editor of the IEEE TRANSACTIONS ON CIRCUITS AND SYSTEMS I and Journal of Signal Processing Systems. He was Chairman of the IEEE Hong Kong Chapter of Signal Processing 2000-2002 and an Organizing Committee Member of the IEEE ICASSP 2003 and ICIP 2010. 